\title{
ON THE EXISTENCE AND UNIQUENESS OF FIXED POINTS FOR HOLOMORPHIC MAPS IN COMPLEX BANACH SPACES
}

\author{
KAZIMIERZ WLODARCZYK
}

(Communicated by Clifford J. Earle, Jr.)

\begin{abstract}
We consider the problem of the existence and uniqueness of fixed points in $X$ of holomorphic maps $F: X \rightarrow X$ of bounded open convex sets $X$ in complex Banach spaces $E$. As a result of the Earle-Hamilton theorem, the problem in the case where $F(X)$ lies strictly inside $X$ (i.e., $\operatorname{dist}[F(X), E \backslash X]>0)$ has a solution. In this article we show that this problem is also solved in the case where $F(X)$ does not lie strictly inside $X$ (i.e., $\operatorname{dist}[F(X), E \backslash X]=0$ ) whenever: (i) $F$ is compact; (ii) $F$ is continuous on $\bar{X}$ and $F(\bar{X}) \subset \bar{X}$; (iii) $F$ has no fixed points on $\partial X$; and (iv) for each $x \in X, 1$ is not contained in the spectrum of $D F(x)$.
\end{abstract}

\section{INTRODUCTION}

Let $X$ be a bounded open convex subset of a complex Banach space $E$. A subset $X^{\prime}$ of $X$ lies strictly inside $X$ if there is an $\varepsilon>0$ such that $\|x-y\| \geq \varepsilon$ whenever $x \in X^{\prime}$ and $y \notin X$.

The old papers of Wolff [30] and Denjoy [4] where $X$ is the open unit disk in $\mathbb{C}$ (see also Burckel [1]); and recent papers of Kubota [15], MacCluer [18], and Chen [2] where $X$ is the open unit ball in $\mathbb{C}^{n}$; Goebel and Reich [7], Hayden and Suffridge [9], Kuczumow and Stachura [16], Stachura [23], Suffridge [24], and Vigue [26] where $X$ is the unit Hilbert ball or the Cartesian product of $n$ unit Hilbert balls; Ky Fan [6] for analytic maps of operators in the sense of functional calculus; and the author $[28 ; 29]$ where $X$ is a homogeneous domain in a $J^{*}$-algebra deal with a few questions concerning the existence of fixed points of holomorphic maps $F: X \rightarrow X$ such that $F(X)$ does not lie strictly inside $X$. In these papers the problem of the uniquenesss of fixed points is not studied. This problem is entirely solved in the case where $F(X)$ lies strictly inside $X$ by Earle and Hamilton [5]:

Theorem 1.1. Let $X$ be a bounded open connected subset of a complex Banach space $E$. If a holomorpic map $F: X \rightarrow X$ is such that $F(X)$ lies strictly inside $X$, then $F$ has a unique fixed point in $X$.

Received by the editors January $25,1990$.

1980 Mathematics Subject Classification (1985 Revision). Primary 46G20, 47H10.

Key words and phrases. Complex Banach spaces, holomorphic maps, bounded open convex sets, fixed points. 
If $E$ is infinite dimensional, then the above condition on $F(X)$ is much weaker than the usual condition of the compact closure in $X$. When $E=\mathbb{C}^{n}$ the two conditions coincide and Theorem 1.1 reduces to Hervé [11, p. 83] and Reiffen [20, p. 322].

This remarkable result is the kernel of the study of the fixed point theory of holomorphic maps. It was extended, applied, and studied by Harris [10, Appendix, pp. 102-104; 8], Hayden and Suffridge [10], Hervé [12], Vesentini [25], and others.

The purpose of his paper is to give a condition that guarantees the existence and uniqueness of fixed points of holomorphic maps $F: X \rightarrow X$ of bounded open convex subsets $X$ of arbitrary complex Banach spaces $E$ in the case where $F(X)$ does not lie strictly inside $X$; i.e., when $\operatorname{dist}[F(X), E \backslash X]=0$. In the proof we use [27], properties of holomorphic maps, and degree theory in complex Banach spaces.

\section{RESUlt}

To state our result, we need some conventions.

A map $F$ is called holomorphic if the Fréchet derivative of $F$ at $x$ (denoted by $D F(x)$ ) exists as a bounded complex linear map for each $x$ in the domain of definition of $F$; see Hille and Phillips [13, Chapters 3, 26] for details.

Let $E$ be an infinite-dimensional complex Banach space. Denote the set of bounded linear operators on $E$ by $B(E)$. Let $\sigma(A)$ denote the spectrum of an element $A$ of $B(E)$.

For a set $X, \bar{X}$ and $\partial X$ stand for the closure of $X$ and the boundary of $X$, respectively. A subset $X^{\prime}$ of $X$ lies strictly inside $X$ if there esists $\varepsilon>0$ such that $x+y \in X$ for all $x \in X^{\prime}$ and $\|y\|<\varepsilon$.

A map $F: X \rightarrow X$ is said to be compact if $F(X)$ is contained in a compact subset of $E$.

Our theorem is as follows:

Theorem 2.1. Suppose $X \subset E$ is a bounded open convex set and $F: X \rightarrow X$ a holomorphic map in $X$ such that $F(X)$ does not lie strictly inside $X$. If (i) $F$ is compact; (ii) $F$ is continuous on $\bar{X}$ and $F(\bar{X}) \subset \bar{X}$; (iii) $F$ has no fixed points on $\partial X$; and (iv) for each $x \in X, 1$ is not contained in $\sigma(D F(x))$, then $F$ has a unique fixed point in $X$.

\section{Proof of Theorem 2.1}

Write $G=I-F, I$ being the identity on $E$. If $\Omega$ denotes the set of all zeros of $G$ in $X$, then $\Omega$ is nonempty by [27] and (iii). It is sufficient to show that $\Omega$ contains one element.

We first show that $\Omega$ is finite. Towards a contradiction, let $x, x_{m} \in \Omega$, $m=1,2, \ldots$, be such that $\left\|x_{m}-x\right\| \rightarrow 0$ as $m \rightarrow \infty$; by the compactness of $F$, the results of [27], and assumptions (i)-(iii), such a point $x$ and a sequence $\left(x_{m}\right)$ exist. Now since the map $u \rightarrow D G(u)$ is a holomorphic map of $X$ into 
$\mathscr{L}(E, E)$ (see Nachbin [19, Proposition 3, p. 29]), by the inverse map theorem for complex Banach spaces (see Rudin [21, p. 243]), the map $G$ is locally biholomorphic in $X$; i.e., in particular, there exists a neighbourhood $U$ of $x$ in $X$ that $G(U)=V$ is open in $E, G^{-1}$ exists, and $G^{-1}$ is holomorphic in $V$. If $m$ is sufficiently large, however, $x_{m} \in U$. This yields a contradiction.

If $\Omega=\left\{x_{1}, x_{2}, \ldots, x_{n}\right\}$ then by Schwartz [22],

$$
\operatorname{deg}(G, X, 0)=\operatorname{deg}(G, X \backslash D, 0)=\sum_{k=1}^{n} \operatorname{deg}\left(G, D_{k}, 0\right),
$$

where $D_{k}$ are small neighbourhoods of $x_{k}$ such that the sets $\bar{D}_{k}$ are pairwise disjoint, $\bar{D}_{k} \subset X$, and $D=\bar{X} \backslash\left(\bigcup_{k=1}^{n} D_{k}\right)$.

Without loss of generality we assume that $0 \in X$. (Otherwise we replace the map $F$ and the set $X$ by the map $F_{0}$ and the set $X-a$, where $F_{0}: X-a \rightarrow$ $X-a$ is defined by $F_{0}(u)=F(u+a)-a, a \in X$ is arbitrary and fixed.)

Since the implications $t F(\bar{X}) \subset \bar{X}$ hold for $0 \leq t \leq 1$, we clearly have

$$
\{0(1-t)+t F(u)\} \cap \partial X=\varnothing \text { for } u \in \partial X \text { and } 0 \leq t<1 .
$$

With (iii) this leads to

$$
\{0\} \cap h_{t}(\partial x)=\varnothing \text { for all } 0 \leq t \leq 1,
$$

where

$$
h_{t}(u)=u-t F(u), \quad u \in \bar{X}, 0 \leq t \leq 1 .
$$

Applying the homotopy property to $h_{t}$ (see Cronin [3], Lloyd [17], Schwartz [22]), we get

$$
\operatorname{deg}(G, X, 0)=\operatorname{deg}(I, X, 0)=1 .
$$

Further, since $E$ is complex, the multiplicity of each eigenvalue of $D F\left(x_{k}\right)$, $k=1,2, \ldots, n$, is even. By Krasnoselskii [14, Theorem 4.7] this gives

$$
\operatorname{deg}\left(G, D_{k}, 0\right)=1, \quad k=1,2, \ldots, n .
$$

From (3.1)-(3.3) we deduce that $n=1$. This completes the proof.

\section{EXAMPLES}

Let $E=\mathbb{C}$ and $X=\{x \in \mathbb{C}:|x|<1\}$.

(a) If $F$ is not assumed to satisfy (iii) or (iv), the result of Theorem 2.1 need not hold. In fact, we have constructed a simple example of $F$,

$$
F(x)=\left(x^{2}+1\right) / 2, \quad x \in X,
$$

that maps $X$ into $X$, where $F(X)$ does not lie strictly inside $X$, and $F$ is nonexpansive and compact in $X$, holomorphic in some neighbourhood of $X$, but has no fixed points in $X$.

(b) Let

$$
F(x)=-(x-c) /(1-\bar{c} x), \quad 0<|c|<1, x \in X .
$$


If $x \in X$ is arbitrary and fixed, then $D F(x) h-h=0$ whenever $h=0$. Thus $F$ satisfies the assumptions of Theorem 2.1. We have

$$
\text { Fix } F=\left\{c\left[1+\left(1-|c|^{2}\right)^{1 / 2}\right]^{-1}\right\} \subset X, \quad 0<|c|<1 .
$$

\section{REFERENCES}

1. R. B. Burckel, Iterating analytic self-maps of disc, Amer. Math. Monthly 88 (1981), 396407.

2. G.-N. Chen, Iteration for holomorphic maps of the open unit ball and the generalized upper half-plane of $\mathbb{C}^{n}$, J. Math. Anal. Appl. 98 (1984), 305-313.

3. J. Cronin, Fixed points and topological degree in nonlinear analysis, Math. Surveys, vol. 11, Amer. Math. Soc., Providence, RI, 1964.

4. A. Denjoy, Sur l'iterarion des fonctions analytiques, C. R. Acad. Sci. Paris 182 (1926), 255-257.

5. C. J. Earle and R. S. Hamilton, A fixed point theorem for holomorphic mappings, Global analysis (Berkeley, Calif., 1968), Proc. Sympos. Pure Math., vol. 16, Amer. Math. Soc., Providence, RI, 1970, pp. 61-65.

6. K. Fan, Iteration of analytic functions of operators, Math. Z. 179 (1982), 293-298.

7. K. Goebel and S. Reich, Uniform convexity, hyperbolic geometry and nonexpansive mappings, Marcel Dekker, New York, 1984.

8. L. A. Harris, Schwarz-Pick systems of pseudometrics for domains in normed linear spaces, Advances in Holomorphy (J. A. Barroso, ed.), North-Holland Math. Stud., vol. 34, Amsterdam, 1979, pp. 345-406.

9. T. L. Hayden and T. J. Suffridge, Biholomorphic maps in Hilbert space have a fixed point, Pacific J. Math. 38 (1971), 419-422.

10. _ Fixed points of holomorphic maps in Banach spaces, Proc. Amer. Math. Soc. 60 (1976), 95-105.

11. M. Hervé, Several complex variables, local theory, Oxford Univ. Press and Tata Inst. of Fund. Research, Bombay, 1963.

12. __ Some properties of the images of analytic maps, North-Holland Math. Stud., vol. 12, Amsterdam, 1977, pp. 217-229.

13. E. Hille and R. S. Phillips, Functional analysis and semigroups, Amer. Math. Soc. Colloq. Publ., vol. 31, Amer. Math. Soc., Providence, RI, 1957.

14. M. A. Krasnoselskii, Topological methods in the theory of nonlinear integral equations, Macmillan, New York, 1964.

15. Y. Kubota, Iteration of holomorphic maps of the unit ball into itself, Proc. Amer. Math. Soc. 88 (1983), 476-480.

16. T. Kuczumow and S. Stachura, Fixed points of holomorphic mappings in the Cartesian product of $n$ unit Hilbert balls, Canad. Math. Bull. 29 (1986), 281-286.

17. N. G. Lloyd, Degree theory, Cambridge Tracts in Math., vol. 73, Cambridge Univ. Press, Cambridge and New York, 1978.

18. B. D. MacCluer, Iterates of holomorphic self-maps of the unit ball in $\mathbb{C}^{N}$, Michigan Math. J. 30 (1983), 97-106.

19. L. Nachbin, Topology on spaces of holomorphic mappings, Springer-Verlag, Berlin, Heidelberg, and New York, 1969.

20. H.-J. Reiffen, Die Carathéodorysche Distanz und ihre zugehörige Differentialmetric, Math. Ann. 161 (1965), 315-324.

21. W. Rudin, Functional analysis, McGraw-Hill, New York, 1973.

22. J. T. Schwartz, Nonlinear functional analysis, Gordon and Breach, New York, London, and Paris, 1969. 
23. A. Stachura, Iterates of holomorphic self-maps of the unit ball in Hilbert space, Proc. Amer. Math. Soc. 93 (1985), 88-90.

24. T. J. Suffridge, Common fixed points of commuting holomorphic maps of the hyperball, Michigan Math. J. 21 (1974), 309-314.

25. E. Vesentini, Invariant distances and invariant differential metric in locally convex spaces, Spectral Theory, Banach Center Publ., no. 8, Polish Scientific, Warsaw, 1982, pp. 493-512.

26. J.-P. Vigué, Points fixes d'applications holomorphes dans un produit fini de boules unités d'espaces de Hilbert, Ann. Mat. Pura Appl. 137 (1984), 245-256.

27. K. Wlodarczyk, Approximative fixed points of compact holomorphic maps in complex Banach spaces, Atti Sem. Mat. Fis. Univ. Modena 36 (1988), 153-157.

28. __ Julia's lemma and Wolff's theorem for $J^{*}$-algebras, Proc. Amer. Math. Soc. 99 (1987), 472-476.

29. __ Studies of iterations of holomorphic maps in $J^{*}$-algebras and complex Hilbert spaces, Quart. J. Math. Oxford 37 (1986), 245-256.

30. J. Wolff, Sur une généralisation d'un theorémè de Schwarz, C. R. Acad. Sci. Paris 182 (1926), 918-920; 183 (1926), 500-502.

INSTitute of Mathematics, University of Lódź, Banacha 22, 90-238 Lódź, Poland 